\title{
Análise dos valores glicêmicos de participantes do Programa de Atendimento ao Idoso - Unochapecó
}

\author{
Suzane Orte Cardoso da Silva \\ Carlla Andréia Henchen \\ Carine Ariéli Grings \\ Gilcimara Ferrazo \\ Mônica Andréia Dal Magro \\ Lilian Marin
}

CADERNO DE RESUMOS

Fisisenectus . Unochapecó Ano 1 - Edição especial - 2013 p. 135

Suzane Orte Cardoso da Silva, acadêmica do curso de Fisioterapia da Universidade Comunitária da Região de Chapecó (Unochapecó), suorte@unochapeco.edu.br

\section{Resumo}

Introdução: Com o aumento da longevidade, o envelhecimento populacional é um fator predisponente ao desenvolvimento de um maior número de doenças e agravos. Sabe-se que $75,5 \%$ dos idosos possuem algum tipo de doença crônica, como, por exemplo, o diabetes mellitus. 0 monitoramento laboratorial do nível glicêmico é importante para acompanhar e prevenir as complicações. 0 diagnóstico em idosos é dificultado devido a mudanças fisiológicas próprias do processo de envelhecimento. Conforme a Sociedade Brasileira de Diabetes, os valores de referência empregados para a taxa de glicose em jejum normal é de 60 a 99 mg $\mathrm{dL}^{-1}$. Objetivo: Analisar os níveis glicêmicos de idosos participantes do Programa de Atendimento ao Idoso. Metodologia: Foram avaliados 36 indivíduos em processo de senescência e idosos, com média de idade $63,75 \pm 8,74$ de ambos os gêneros, com predomínio do gênero feminino, não praticantes de atividade física regular. Utilizou-se para isso glicosímetro e fitas de aferição glicêmica da marca Onetouch Ultra 2, agulhas descartáveis da marca ACCU-CHEK, algodão, álcool 70\% e folha de resposta. Todos os indivíduos estavam em média por duas horas de jejum, estes tiveram a sua polpa digital do seu quarto quirodáctilo puncionado com agulha, sendo que estes foram assepsiados com algodão e álcool 70\%, posteriormente foi coletada uma gotícula de sangue em fita glicêmica para aferição da glicose capilar. Utilizou-se como valor de referência da glicose plasmática de jejum forem $\geq 126 \mathrm{mg} / \mathrm{dl}$ ou $\geq 110$ e $<126 \mathrm{mg} / \mathrm{dl}$ e na presença de dois ou mais fatores de risco para DM nos indivíduos com idade $\geq 45$ anos. Parâmetros do Consenso Brasileiro de Diabetes do ano de 2000. Resultados: Após as aferições verificou-se que estes apresentaram níveis glicêmicos médios de $100,58 \pm 25,20$. Ressalta-se que o menor nível glicêmico foi de $78 \mathrm{mg} / \mathrm{dl}$ e o maior foi de $204 \mathrm{mg} / \mathrm{dl}$ e, no entanto, nenhum era portador de diabetes mellitus. Considerações finais: Apesar dos indivíduos não realizarem atividade física regular, estes não apresentaram níveis glicêmicos médios alterados, contudo é importante o controle glicêmico no processo de envelhecimento, já que a diabetes mellitus é uma doença que está intimamente interligada com esta população. Importante evidenciar que a fisioterapia possui uma ampla atuação tanto com indivíduos diabéticos quanto em idosos, tanto na promoção, prevenção quanto na reabilitação. Porém há necessidade de maior conscientização desta população não apenas no controle de dieta alimentar, mas também na prática regular de atividade física, para manter os valores glicêmicos dentro dos parâmetros de normalidade e evitar doenças crônicas como a diabetes.

\section{Palavras-chave}

Glicemia. Idoso. Fisioterapia. 